\title{
AUTOMATED DIGITAL ODONTOMETRIC STUDY OF MANUAL AND COMPUTER- AIDED METHODS OF TOOTH CROWN MODELLING IN DENTISTRY
}

\author{
A.V. Gaboutchian ${ }^{1 *}$, V. A. Knyaz ${ }^{2,3}$, S. Apresyan ${ }^{4}$ \\ ${ }^{1}$ Moscow State Medical-Stomatological University, Moscow, Russia - armengaboutchian@ mail.ru \\ ${ }^{2}$ State Research Institute of Aviation System (GosNIIAS), 125319 Moscow, Russia - knyaz@gosniias.ru \\ ${ }^{3}$ Moscow Institute of Physics and Technology (MIPT), Russia \\ ${ }^{4}$ Peoples Friendship University of Russia, 117198, Moscow, Russia - dr.apresyan@ gmail.com
}

Commission II, WG II/10

KEY WORDS: Automated Digital Odontometry, Photogrammetry, Tooth Crown, Odontometry, Tooth Crown Modelling

\begin{abstract}
:
One of the most widely used methods of prosthetic treatment in dentistry is restoration of damaged teeth by artificially fabricated crowns, which, regardless of technological variations, always pass through modelling stage. The main two methods of restoration modelling are traditionally used manual and becoming more advanced and widespread computer-aided, or digital. A similar situation is observed in odontometry - tooth measurements - where manual and digital approaches meet, fuse and new methods, as automated digital odontometry, appear. In this study the measurement method is applied to crowns modelled by different dental technicians on trial basis for teeth, prepared on stone cast model of complete dental arches. The same plaster models, before and after tooth preparations, as well as after modelling tooth crowns manually, were scanned for comparative and modelling (this time using computer-aided techniques) reasons. The obtained 3D images were used for measurements by digital automated odontometry to estimate differences of modelled crowns with initial shape of intact, subjected to experimental preparation and restoration, tooth. Comparisons were made within each, manual and digital, crown modelling groups as well; thus comparisons of proposed on default by professional dental software crown shapes were made, and in line with it the influence of different technicians adjustments on modelling precision was estimated. At the same time the measurement method was tested as well by determining the most characteristic differentiating odontometric parameters for the study, which is held largely due to and on the basis of photogrammetric techniques.
\end{abstract}

\section{INTRODUCTION}

Traditional restoration modelling in dentistry is based on applying wax or other materials on models or frameworks fabricated of hard materials, such as plaster, plastic, metal or zirconia. There is a variety of techniques and recommendations describing the process of restoration shaping, for instance wax addition (Mulay, 2011), (Smith and Howe, 2006); (Shillingburg et al., 2000). They are mainly based on knowledge of dental morphology and use of sculptor skills; objective crown shape analysis methods, such as measurements, even if applied, are narrowly limited (Dmitrienko et al., 1998). Professional dental CAD/CAM systems are based on programming methods; they propose tooth crown shapes, usually from digital libraries, which are modified by dental technician through software utilities. There are certainly multiple factors described in dental theory and practical guides impacting results, functional or aesthetic, of dental treatment. And visually those results appear to be satisfying on the modern level of dental technology development. However we are interested in seeking new objective estimates, beyond capabilities of our optical analyser, referring to shapes and morphology of teeth or restorations. Such opportunities are provided digital technology as well and are proposed as automated digital odontometry, which in fact originates from studies in dentistry.

Briefly, this odontometric method is based on measurements on $3 \mathrm{D}$ images of teeth and includes automated detection of tooth axis, automated odontotomy (slicing tooth crown) and automated measurements on the obtained contours of teeth. In comparison with recognized traditional methods (Zubov, 2006), the automated digital ones propose higher variability and multiplicity of parameters, measurement objectivity and decrease, especially referring to the obtained data volume, study time consumption. Such properties have determined the choice of the odontometric method in the current research which has a comparative and experimental outline. Thus modelled restorations are compared to intact tooth shape, which serves as a benchmark. It should be mentioned that in clinical practice intact teeth are usually not covered by crowns and any data dealing with the original tooth shape, is lost in the majority of cases to the moment of treatment necessity. Referring to the current study, in line with morphology of intact teeth, manual and digital crown modelling are assessed by means of objective methods. This becomes possible through combination of experimental preparation of teeth on stone cast models and their scanning on key stages of crown fabrication technology. Though automated digital odontometry provides numerous measured and calculated parameters, the most widely accepted are assessed in this pilot study. It is worth noting that multiple subjective factors exist, such as individual features of dental technicians, which influence on modelling. But more extensive research is necessary for providing a sufficient data amount for this kind of study. Dealing with digital method, professional modelling systems provide default tooth crown outlines further reshaped by technicians. Here our study interests are in measuring modelled crowns in both conditions, before and after reshaping.

\footnotetext{
${ }^{*}$ Corresponding author
} 
It should be mentioned that odontometric studies are conducted on 3D models in dentistry; they support mainly traditional tooth and arch measurement techniques accepted in anthropological or orthodontic studies.

\section{RELATED WORK}

Odontometric studies are carried out for various applications in dentistry, for instance in orthodontics (Chate, 2012), (Naidu and J Freer, 2013a) or anthropology (Townsend et al., 2012), with the application of traditional manual measurement techniques. Though implementing of 3D digital models and appropriate software expands odontometric research opportunities in multiple directions, starting from many conveniences in research conduction to unreachable before image processing prospects. As a support of technological innovations in tooth measurements various types of dental laboratory or similar scanners are found to be suitable for studies, including measurements on separate teeth (Jacob et al., 2015). Reliability of images obtaining equipment and has been assessed in different combinations of intraoral, optical, laser or CT scanners (Al-Rawi et al., 2010), (Rajshekar et al., 2017), (Sang et al., 2016).

As we mentioned before traditional manual measurement techniques, being used on real objects, are sometimes duplicated on 3D images (Nouri et al., 2009), (Kazzazi and Kranioti, 2017). In some cases geometric morphometrics are applied for tooth shape analysis (Gomez-Robles et al., 2007); other authors suggest diversifying approaches to measurements (Smith et al., 2009). More profound in terms of tooth structure analysis are recent studies implementing statistical shape modelling (Woods et al., 2017).

Scanning and measurements involve, depending on the study nature, different object: dental arch casts, mandibles, separate teeth. A significant part of odontometric research in dentistry relates to orthodontics (Naidu and J Freer, 2013b), but usually not artificially fabricated crown, as done in the current study.

Occlusal surfaces of distal teeth premolars and molars has been a subject of interest for various studies in dentistry (Wang et al., 2004), (Gaboutchian et al., 2018) or anthropology (GomezRobles et al., 2007), (Takayama et al., 2012) because of its high importance in functional, evolutional, clinical and other aspects. The current study as well deals with study of odontometric parameters related to occlusal surface of teeth and their artificially fabricated crowns.

\section{METHODOLOGY}

The study is conducted, due to implementing photogrammetric and automated digital odontometric methods, on 3D models of dental arches, teeth, prepared teeth and modelled crowns. The initial stage of the research was in obtaining 3D images of complete dental arches (upper and lower), which included potential application of traditionally used sequence: silicone impression plaster model scanning, or direct intraoral scanning, both providing sufficient image quality and precision. The nature of the research necessitated the use of hard copies of dental arches for tooth preparation and modelling reasons. Thus the first, traditional method of model obtaining was chosen and stationary laboratory scanning units were used (Evolution plus, Zfx; S600 Arti Scanner, ZirkonZahn) and custom designed photogrammetric system at GosNIIAS (Knyaz and Zheltov, 2008).
Models of dental arches were scanned prior to undertaking any procedures and manipulations with them (Figure 1). The scan was used for measurements of morphological features on studied teeth in their intact condition and further comparisons with modelled crowns. The same models were used for tooth preparation usually clinical procedure of providing sufficient shape of tooth for proper crown fabrication, insertion and retention.
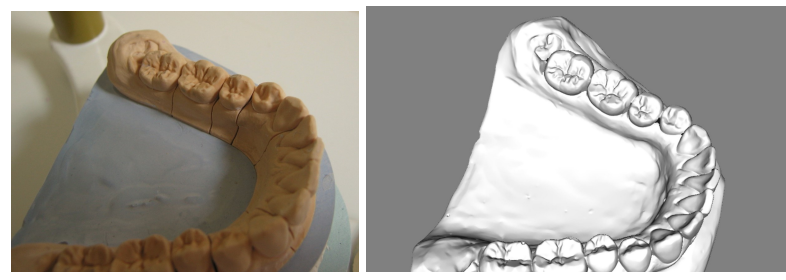

Figure 1. Stone cast model of the lower dental arch (left) and its 3D image (right)

Preparation was conducted in accordance with accepted in dentistry techniques. However experimental nature of the study and medical ethical standards do not allow this kind of studies on real patients, and preparation was performed on plaster model. Prepared teeth were scanned within dental arches as well as separately (Figure 2).
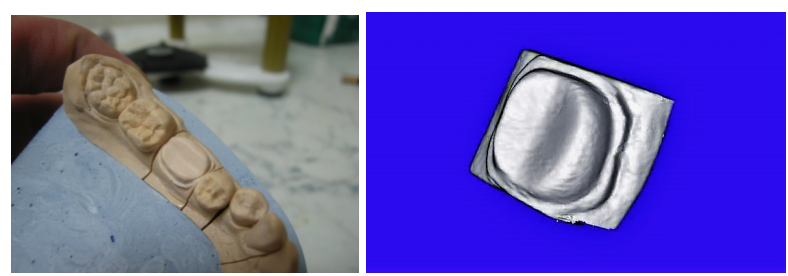

Figure 2. Prepared tooth on stone cast model of the lower dental arch and its 3D image

Prepared teeth provided for starting modelling stage of the study, and the initial phase is wax modelling performed directly on stone cast models ( Figure 3). Wax modelling was performed manually in accordance with accepted dental technical methods.
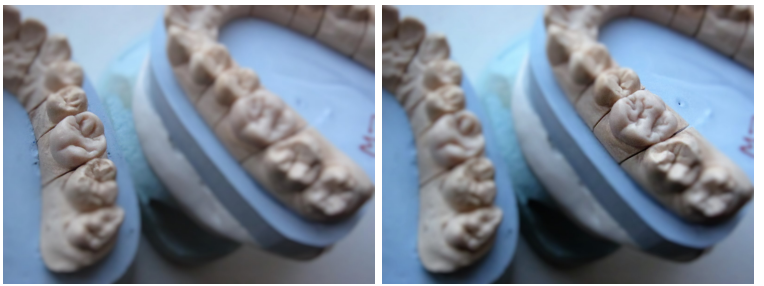

Figure 3. Modelled crowns on stone casts, ivory wax

Dental technicians were not provided with models or images of intact teeth in order to bring the modelling process nearer to real conditions. Modelled crowns were certainly scanned including separate scanning and scan of dental arches as well (Figure 4).

Digital method of modelling was the next step in obtaining crown prototypes and, at the same time, 3D images for the current study. Two stages in the process of digital modelling were fixed through image saving for further measurements: tooth crown shape suggested by default from the library and the technicians final reshaping result (Figure 5).

Scanning process was followed by measurements conducted by means of automated digital odontometry. The method is based 


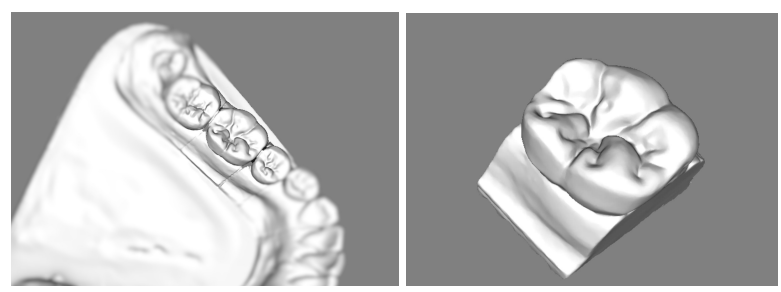

Figure 4. Wax crown scan on stone casts of dental lower arch and separate tooth
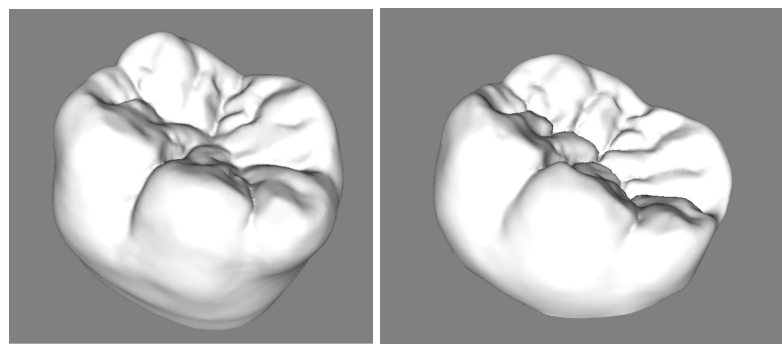

Figure 5. Crowns modelled digitally by default (left) and after reshaping (right)

on multidirectional odontotomic sectioning of teeth: with radial semi-planes and equally spaced parallel planes, which automatically slice studied teeth (Figure 6).
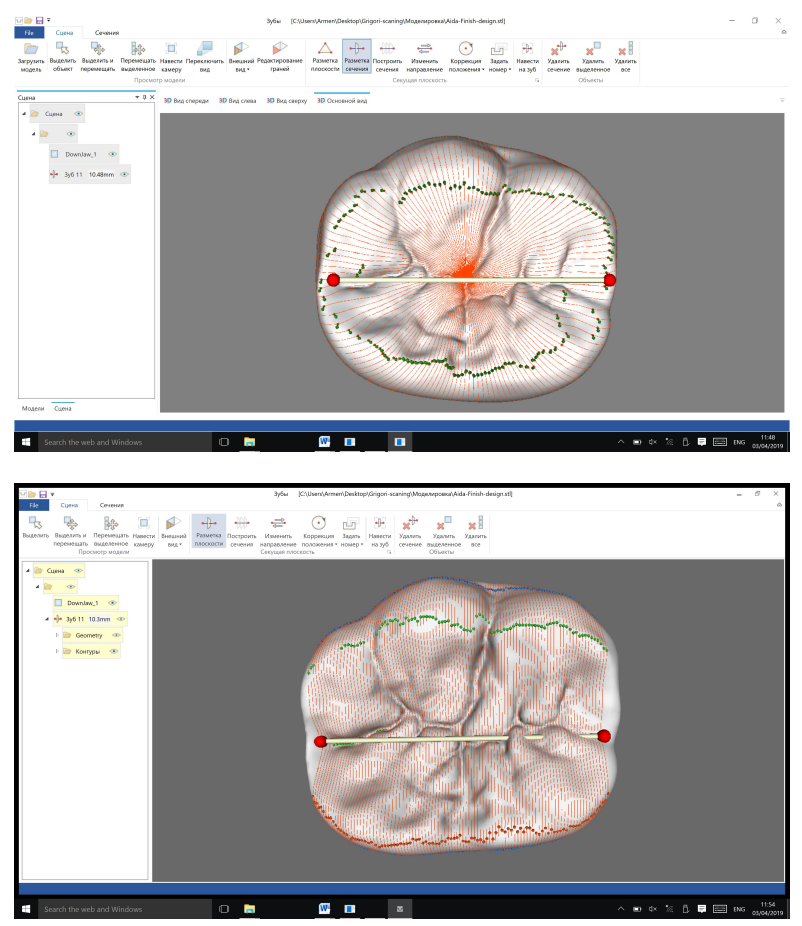

Figure 6. Multidirectional digital modelled crown sectioning

As our previous studies has showed that sectioning direction influences the results, the current version of odontometric software provides automated detection of sectioning direction together with tooth axis alignment. Sections containing contours of tooth crowns serve for automated measurements including obtaining the odontometric parameters dealing with the current study (Figure 7).

Without focusing on measurement technique details (as it has been described earlier), we would like to mention that current odontometric research, due to software improvements, has been held in fully automated mode. Another characteristic feature of
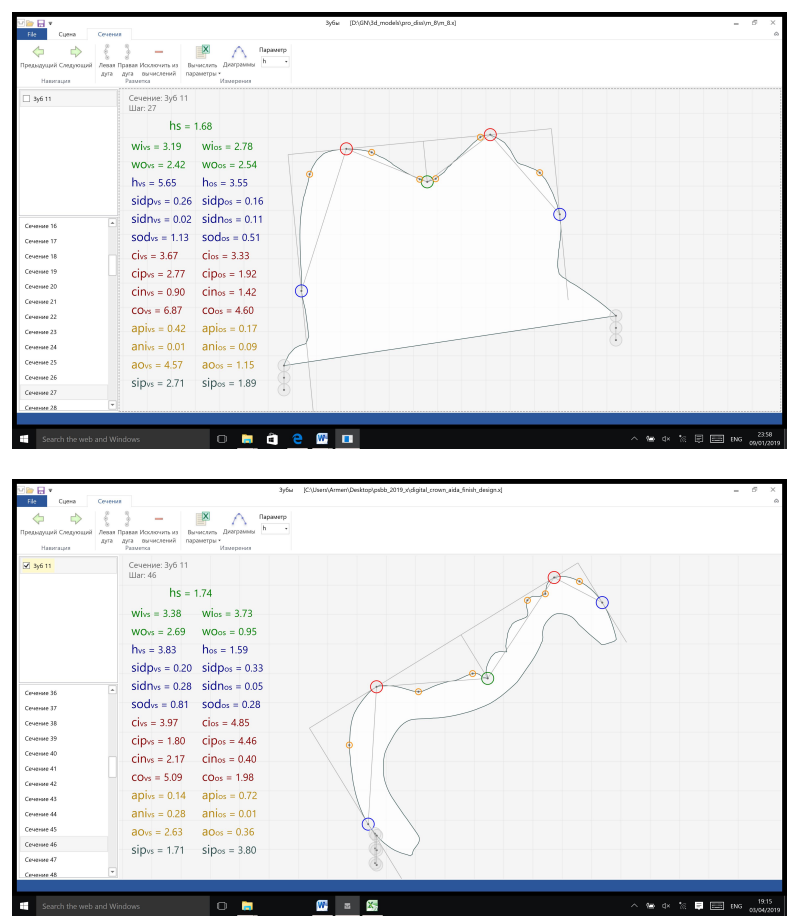

Figure 7. Tooth (upper) and crown (lower) contours with measurement outlines and parameters

the current study is in sequential use of same plaster models for all steps of the study which allows precise superimposing of 3D images corresponding to every preliminary or modelling stage. Thus we achieve ability to minimise data distortion as odontometric parameters can be obtained on similar sections for all samples of modelled crowns corresponding to each studied tooth. Odontometric parameters, and therefore modelled crowns, were compared by estimating deviation from similar parameters on intact tooth.

\section{RESULTS AND DISCUSSION}

Crown modelling in dentistry, especially referred to manual method, is a combination of dental system morphology knowledge and manual skills, experience and practice, observations and repeated attempts. Though 3D digital techniques of modelling seem to be more impersonal, they possess a certain degree of subjectivity as well: starting from choice of default crown shape from library content to the process of modelling itself. Thus, despite undeniable usability of digital methods, comparative measurements of modelled crowns are of interest in dentistry, especially taking into consideration their automation degree.

Without expanding on multiplicity of parameters provided by automated digital odontometry, the current study involves basic measurements in vestibular-oral direction. All studied teeth occupy distal parts of dental arches; those teeth are confined, being first molars and second premolars, by neighbouring teeth from mesial and distal sides. Thus their maximal vestibular-oral dimensions were measured (Table 1) as initial parameters for further comparison with manually and digitally modelled crowns.

Similar measurements were conducted on the following sequence of modelled crowns 3D images: manually modelled crown, crown, picked by default from the digital library, and digitally modelled crown. An example of measurement series, referring to the abovementioned sequence is presented in (Table 2). As it can be seen, 


\begin{tabular}{||c|c||}
\hline Tooth Number & Vest-Or max, mm \\
\hline \hline 2.6 & 10.986 \\
3.6 & 9.609 \\
2.5 & 9.736 \\
4.5 & 7.627 \\
1.6 & 11.681 \\
3.6 & 10.337 \\
2.5 & 8.97 \\
4.5 & 8.141 \\
1.6 & 11.52 \\
3.6 & 10.306 \\
4.5 & 8.466 \\
1.6 & 11.359 \\
\hline
\end{tabular}

Table 1. Maximal vestibular-oral dimensions of teeth

regardless of modelling method applied, all crowns show higher results on maximal vestibular-oral dimension if compared to initial, intact tooth crown parameters; though this might mean insufficient tooth preparation, which can be additionally studied.

\begin{tabular}{||ccc||}
\hline Parameter & $\begin{array}{c}\text { Intact tooth } \\
\mathrm{mm}\end{array}$ & $\begin{array}{c}\text { Manual modelling } \\
\mathrm{mm}\end{array}$ \\
\hline \hline Vest-Or max, mm & 10.337 & 11.313 \\
\hline & Digital modelling & \\
\hline & Crown by default & Final crown \\
\hline Vest-Or max, mm & 10.936 & 10.935 \\
\hline
\end{tabular}

Table 2. Maximal vestibular-oral dimensions measured on teeth and crowns

Digital modelling method shows noticeably closer results to initial tooth parameters and consistency in measurements, which actually coincide by value, on both relevant samples; though in fact locations of taken maximal measurements on both crowns do not completely match (Figure 8).

\section{CONCLUSION}

Automated digital odontometry, providing conditions for measurements of teeth or their artificially designed crowns, has a potential for conducting more meticulous studies of tooth morphology than traditional manual odontometric methods.

Digital method of crown modelling can be considered more accurate and preferable in various terms of their usability. However more expanded studies are preferable for diverse morphological analysis, as only limited data volume was currently evaluated for revealing differences in modelling of artificially designed tooth crowns for prosthetic dental treatment. Nevertheless the assessment method is based on objectively measured odontometric parameters and their comparison.

\section{ACKNOWLEDGEMENTS}

The work was performed with the support by Grant 17-29-04509 of Russian Foundation for Basic Research (RFBR).

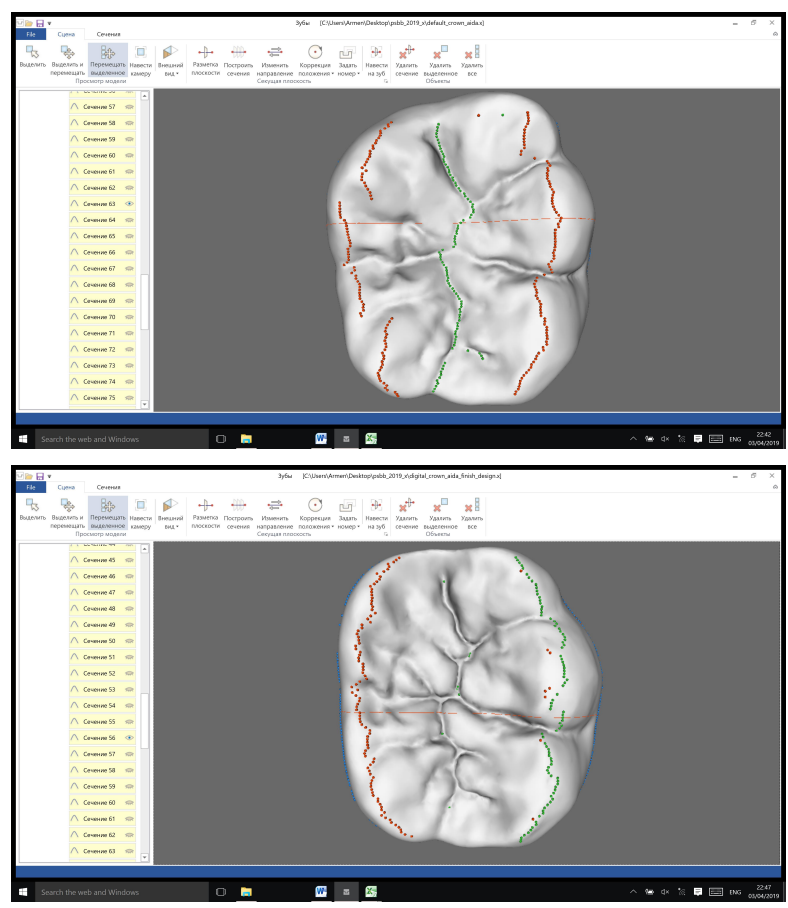

Figure 8. Vest-Or max sections on default (left) and reshaped (right) digital crowns

\section{REFERENCES}

Al-Rawi, B., Hassan, B., Vandenberge, B. and Jacobs, R., 2010. Accuracy assessment of three-dimensional surface reconstructions of teeth from cone beam computed tomography scans. Journal of oral rehabilitation 37, pp. 352-8.

Chate, R. A. C., 2012. Odontometric evaluation of mandibular premolars with tooth shape deviation: A case-control study. The Angle orthodontist 82, pp. 785-91.

Dmitrienko, S., Krayushkin, A. and Ivanov, L., 1998. Tooth modelling guide.

Gaboutchian, A., Simonyan, H., Knyaz, V., Petrosyan, G., TerVardanyan, L., Leybova, N. A. and Apresyan, S. V., 2018. Automated shape analysis of teeth from the archaelogical site of nerqin naver. ISPRS - International Archives of the Photogrammetry, Remote Sensing and Spatial Information Sciences XLII-2, pp. 339345.

Gomez-Robles, A., Martinn-Torres, M., Bermdez de Castro, J.M., Margvelashvili, A., Bastir, M., Arsuaga, J., Prez-Prez, A., Estebaranz-Snchez, F. and Martnez Martnez, L., 2007. A geometric morphometric analysis of hominin upper first molar. Journal of human evolution 53, pp. 272-85.

Jacob, H., D. Wyatt, G. and Buschang, P., 2015. Reliability and validity of intraoral and extraoral scanners. Progress in Orthodontics.

Kazzazi, M. and Kranioti, E., 2017. Applicability of 3d-dental reconstruction in cervical odontometrics. American Journal of Physical Anthropology.

Knyaz, V. and Zheltov, S., 2008. Photogrammetric techniques for dentistry analysis, planning and visualization. ISPRS - International Archives of the Photogrammetry, Remote Sensing and Spatial Information Sciences XXXVII-B5, pp. $783-788$.

Mulay, G., 2011. Waxing techniques to develop proper occlusal morphology in different occlusal schemes. The Journal of Indian Prosthodontic Society 11, pp. 205-209. 
Naidu, D. and J Freer, T., 2013a. The evidence supporting methods of tooth width measurement: Part i. vernier calipers to stereophotogrammetry. Australian orthodontic journal 29, pp. 159-163.

Naidu, D. and J Freer, T., 2013b. The evidence supporting methods of tooth width measurement: Part ii. digital models and intraoral scanners. Australian orthodontic journal 29, pp. 164-9.

Nouri, M., Massudi, R., Akbarzadeh, A., Azimi, S. and Fereidouni, F., 2009. The accuracy of a 3-d laser scanner for crown width measurements. Australian orthodontic journal 25, pp. 417.

Rajshekar, M., Julian, R., Williams, A.-M., Tennant, M., Forrest, A., Walsh, L., Wilson, G. and Blizzard, L., 2017. The reliability and validity of measurements of human dental casts made by an intra-oral 3d scanner, with conventional hand-held digital callipers as the comparison measure. Forensic Science International.

Sang, Y.-H., Hu, H.-C., Lu, S.-H., Wu, Y., Li, W.-R. and Tang, Z.-H., 2016. Accuracy assessment of three-dimensional surface reconstructions of in vivo teeth from cone-beam computed tomography. Chinese Medical Journal 129, pp. 1464.

Shillingburg, H. T., Wilson, E. L. and Morrison, J. T., 2000. Guide to occlusal waxing. 3 rd ed.

Smith, E. and Howe, L., 2006. Planning and Making Crowns and Bridges. CRC Press.

Smith, R., Zaitoun, H., Coxon, T., Karmo, M., Kaur, G., Townsend, G., F Harris, E. and Brook, A., 2009. Defining new dental phenotypes using 3-d image analysis to enhance discrimination and insights into biological processes. Archives of oral biology 54 Suppl 1, pp. S118-25.

Takayama, H., Kato, H. and Townsend, G., 2012. The main occluding area between opposing teeth during chewing: A comparison between australians and japanese. New Directions in Dental Anthropology: Paradigms, Methodologies and Outcomes pp. 106-114.

Townsend, G., Kanazawa, E. and Takayama, H., 2012. New directions in dental anthropology : paradigms, methodologies and outcomes / edited by Grant Townsend, Eisaku Kanazawa [and] Hiroshi Takayama. University of Adelaide Press Adelaide.

Wang, M.-Q., Zhang, M. and Zhang, J.-H., 2004. Photoelastic study of the effects of occlusal surface morphology on tooth apical stress from vertical bite forces. The journal of contemporary dental practice 5, pp. 74-93.

Woods, C., Ferne, C., Browne, M., Zakrzewski, S. and Dickinson, A., 2017. The potential of statistical shape modelling for geometric morphometric analysis of human teeth in archaeological research. PLOS ONE.

Zubov, A. A., 2006. Methodological handbook on anthropological analysis of odontological materials. 